Objectives: To assess the use and drivers of SLEDAI and BILAG index in real world clinical practice.

Methods: A cross-sectional study of rheumatologists in the US and EU5. Data were collected from the Adelphi Real World 2010/2013/2015 Lupus Disease Specific Programmes (DSP). Physicians were asked to complete an attitudinal survey and patient record forms (PRFs) for the next 5 patients consulting with SLE; the same patients were asked to complete patient self-completion (PSC) forms describing how SLE affected them PRFs collected data pertaining to the patient's diagnosis, disease history, current clinical outcomes, treatment and management history. PSCs focused on similar data collection and included patient reported outcome measures (PROs) to assess the humanistic burden. 2015 DSP data was used to assess the profile of patients with a SLEDAI assessment were compared to those without a SLEDAI assessment, using Fisher's Exac and Mann-Whitney tests. Similar analysis was also conducted for those with/without BILAG index assessments. 2013 \& 2010 DSP datasets were merged, and multiple linear regression was used to assess drivers of SLEDAI and BILAG index utility, respectively.

Results: Physicians provided 263 surveys, extracted from the 2015 DSP, indicating that 131 were aware of but did not use the BILAG index, and 92 of physicians were aware of but did not use the SLEDAI.

Physicians provided 1376 record forms for SLE patients, extracted from the 2015 DSP; 71 (5.2\%) had a BILAG index (1305, 94.8\% had no BILAG index), and $373(27.1 \%)$ had had a SLEDAl calculated prospectively $(1003,72.8 \%$ had No SLEDAI). Patients with SLEDAl had longer disease duration that patients who did not have a SLEDAI (mean: 6.3 vs. 5.2 years, $p=0.007$ ), were less likely to have been described as mild at diagnosis (no SLEDAI mild: 18.7\%; SLEDAI mild: $11.3 \%, p=0.0004$ ) and consulted more with health care professionals in the past 12 months (no SLEDAI mean visits: 6.5, SLEDAI mean: 7.7, $\mathrm{p}<0.001$ ).

Patients with a BILAG index had flared more in the last 12 months (no BILAG: $30.6 \%$, BILAG: $45.1 \%, p=0.0126$ ).

Physicians provided data on 220 SLEDAI and 75 BILAG assessments, extracted from the 2010 \& 2013 DSPs. Multiple linear regression analyses revealed that flaring in the last 12 months (Coef: 3.27 [0.36 - 6.18], $\mathrm{p}=0.028$ ), renal symptoms (Coef: 5.67 [1.84-9.51], $\mathrm{p}=0.004$ ), and muscular symptoms (Coef: 4.58 [1.22-7.94], $\mathrm{p}=0.008$ ) were all associated with a higher SLEDAI score, with r-squared 0.1948 .

Multiple linear regression showed that renal symptoms (Coef: 3.87 [1.00 - 6.75], $\mathrm{p}=0.009$ ) were associated with a higher BILAG score, with $\mathrm{r}$ squared 0.3717

Conclusion: The use of SLEDAI and BILAG index in clinical practice is limited and seemingly reserved for use in more severe patient cohorts; understanding the ongoing impact of this selective use on the treatment and management of SLE would be beneficial. Additionally, understanding the drivers and barriers to the use of disease activity metrics is important to improve the management of SLE in the future.

Disclosure of Interests: Karen Costenbader: None declared, Ben Hoskin Employee of: Adelphi, Christian Atkinson Employee of: Adelphi, David Bell Employee of: Adelphi, Olivia Massey Employee of: Adelphi Real World, Jennifer H. Lofland Employee of: Janssen Global Commercial Strategic Organization, Pam Berry Shareholder of: GSK and Janssen Global Services, Chetan Karyekar Shareholder of: J\&J, Employee of: Janssen Scientific Affairs, LLC, Abbott, BMS, Novartis, Zahi Touma Grant/research support from: GSK Canada, Consultant for: UBC, Pfizer, Janssen, Inc DOI: 10.1136/annrheumdis-2019-eular.4239

\section{FRI0257 PROLACTIN AND DEHYDROEPIANDROSTERONE SULFATE IN WOMEN WITH ACTIVE SYSTEMIC LUPUS ERYTHEMATOSUS OF RECENT ONSET VERSUS CHRONIC INACTIVE PATIENTS}

Olga Lidia Vera Lastra ${ }^{1}$, Enzo Christopher Vazquez Jimenez ${ }^{1}$, Maria del Pilar Cruz Dominguez ${ }^{1}$, Luis Javier Jara ${ }^{2} .1$ Mexico City, Internal Medicine, Mexico City, Mexico; ${ }^{2}$ Unidad Medica de Alta Especialidad Hospital de Especialidades Dr Antonio Fraga Mouret Centro Medico Nacional La Raza, Direccion de investigacion y educacion, Mexico City, Mexico

Background: Systemic lupus erythematosus (SLE) is an autoimmune disease, typically affecting young women at reproductive age. Prolactin (PRL) levels have shown direct correlation with clinical and serological disease activity. Hyperprolactinemia has been reported in a wide range of SLE patients (15-33\%). Dehydroepiandrosterone (DHEA) and its sulfated form dehydroepiandrosterone sulfate (DHEAS) are the most abundant circulating steroid hormones in humans. The level of both metabolites is lower in patients with active SLE compared with patients with inactive disease, which suggests a potential role of low levels of DHEAS in the pathogenesis of SLE.

Objectives: To evaluate serum PRL levels and DHEAS levels in patients with active SLE of recent onset vs patients with inactive chronic and healthy controls and their correlation with activity and chronicity scores Methods: Serum PRL levels and DHEAS levels were studied, as well as their correlation with SLEDAI and SLICC scores. Group 1: 15 patients with SLE of recent onset (active SLEDAI >3) Group 2: 20 patients with inactive chronic SLE (SLEDAl<3) and Group 3: 20 healthy controls. SLEDAI and SLICC were calculated in group 1 and group 2 respectively. PRL and DHEAS was measurement by radioimmunoassay in all groups. Statistical analysis: U-Mann Whitney and Spearman correlation.

Results: Group 1: serum PRL levels were $27.82 \pm 9.96 \mathrm{ng} / \mathrm{dl}$ vs group 2 $20 \pm 5.02 \mathrm{ng} / \mathrm{dl}(p=0.004)$ and group $3: 19.58 \pm 4.57 \mathrm{ng} / \mathrm{dl}(p=0.004)$. Group 1: serum DHEAS levels were $14.58 \pm 9.26 \mu \mathrm{g} / \mathrm{dl}$, group 2: $19.36 \pm 7.21 \mu \mathrm{g}$ dl $(p=0.04)$ and group 3: $154.43 \pm 50.88 \mu \mathrm{g} / \mathrm{dl}(\mathrm{p}=0.001)$. The average DHEAS was lower in patients with chronic SLE vs controls $(p=0.001)$ A positive linear correlation was found between serum $P R L$ levels and SLEDAI score (Rho 0.92, $\mathrm{p}=0.001$ ). No correlation was found between $\mathrm{PRL}$ and SLICC score. A negative linear correlation was shown between DHEAS concentration and SLICC score (Rho-0.46, $p=0.03$ ).

Conclusion: PRL levels were higher in active SLE patients VS. chronic inactive SLE and healthy controls. In contrast, serum DHAS levels were lower in patients with active SLE vs. inactive chronic SLE. We found a positive correlation between SLEDAl score and PRL serum concentrations and an inverse correlation between SLICC score and DHAS serum levels.

\section{REFERENCES:}

[1] Borba VV, Zandman-Goddard G, Shoenfeld Y. Prolactin and Autoimmunity. Front Immunol 2018; 12:73.

[2] Rutkowski K, Sowa P, Rutkowska-Talipska J, et al. al. Dehydroepiandrosterone (DHEA): hypes and hopes Drugs. 2014;74:1195-207.

[3] Robeva R, Tanev D, Andonova S, et al. Androgen receptor (CAG)n polymorphism and androgen levels in women with systemic lupus erythematosus and healthy controls. Rheumatol Int. 2013;33:2031-8.

Disclosure of Interests: None declared

DOI: 10.1136/annrheumdis-2019-eular.7447

\section{FRI0258 ANTI-RO/SSA POSITIVITY, ANTI-AQUAPORIN4 ANTIBODIES AND CENTRAL NERVOUS SYSTEM INVOLVEMENT: A RETROSPECTIVE STUDY ON A CONTROVERSIAL CORRELATION}

Erika Zecca ${ }^{1}$, Mattia Bellan ${ }^{1,2,3}$, Domizia Vecchio ${ }^{3}$, Cristoforo Comi ${ }^{3}$, Daniele Soddu ${ }^{1}$, Alessandro Croce ${ }^{1}$, Mario Pirisi ${ }^{1,2,3}$, Pierpaolo Sainaghi ${ }^{1,2,3}{ }^{1}{ }^{1}$ A.O. U Maggiore della Carità, Internal Medicine Division, Immunorheumatology Unit, novara, Italy, ${ }^{2}$ Interdisciplinary Center of Autoimmune Diseases- IRCAD, Internal Medicine Division, Immunorheumatology Unit, Novara, Italy; ${ }^{3}$ Università del Piemonte Orientale - UPO, Department of Translational Medicine, Novara, Italy

Background: Different neurological manifestations have been observed in $20-25 \%$ of patients affected by Sjögren's syndrome (SS). Among them, CNS demyelinating diseases, Neuromyelitis optica (NMO) and NMO spectrum disorders (NMOSD) with anti-aquaporin4 antibodies (anti-AQP4) positivity have been described.

Objectives: The aim of the present study was to assess the clinical charachteristics and seroimmunological correlations in patients with RoSSA antibodies and Central and peripheral nervous system (CNS and PNS) involvement.

Methods: We retrospectively reviewed clinical records, laboratory and Magnetic Resonance Imaging (MRI) reports of patients followed-up at a tertiary level immunorheumatology and neuroimmunology clinic. We included patients showing an anti-SSA antibodies positivity and concomitant neurological symptoms at diagnosis. We excluded patients fulfilling SLICC criteria for SLE. We recorded clinical and laboratory and MRI data for all patients.

Results: Out of 9598 clinical records reviewed, we identified 511 patients with anti Ro/SSA positivity. 11 patients had prevalent neurological manifestations. $8(72.7 \%)$ patients were women. The median age was 56 [IQR 31] years. CNS involvemente was the main clinical feature in 7 patients $(63.6 \%) ; 3$ of them $(27.3 \%)$ also had PNS manifestations. 4 patients showed exclusively PNS involvement.

3 subjects fulfilled criteria for SS while 8 patients were classified as undifferentiated connective tissue disease. 7 patients underwent spinal tap 